\section{SIc35a1 deficiency causes thrombocytopenia due to impaired megakaryocytopoiesis and excessive platelet clearance in the liver}

\author{
Xiaolin Ma, ${ }^{1,2,3^{*}}$ Yun $\mathrm{Li}^{1,2^{*}}$ Yuji Kondo, ${ }^{4^{*}}$ Huiping Shi, ${ }^{1,2,4}$ Jingjing Han,,$^{1,2}$ \\ Yizhi Jiang, ${ }^{1,2}$ Xia Bai, ${ }^{1,2,5}$ Stephanie A. Archer-Hartmann, ${ }^{6}$ Parastoo Azadi, ${ }^{6}$ \\ Changgeng Ruan, ${ }^{1,2,5}$ Jianxin Fu ${ }^{1,4,7, \#}$ and Lijun Xia ${ }^{1,2,4, \#}$
}

\begin{abstract}
${ }^{1}$ Jiangsu Institute of Hematology, NHC Key Laboratory of Thrombosis and Hemostasis, The First Affiliated Hospital of Soochow University, Suzhou, China; ${ }^{2}$ Collaborative Innovation Center of Hematology, Soochow University, Suzhou, China; ${ }^{3}$ Department of Hematology, The Affiliated Hospital of Qingdao University, Qingdao, China; ${ }^{4}$ Cardiovascular Biology Research Program, Oklahoma Medical Research Foundation, Oklahoma City, OK, USA; ${ }^{5}$ State Key Laboratory of Radiation Medicine and Protection, Soochow University, Suzhou, China; ${ }^{6}$ Complex Carbohydrate Research Center, University of Georgia, Athens, GA, USA and 'Department of Hematology, The First Affiliated Hospital of Nanjing Medical University, Nanjing, China
\end{abstract}

"XM, YL and YK contributed equally as co-first authors.

"JF and LX contributed equally as co-senior authors.

\section{ABSTRACT}

S ialic acid is a common terminal residue of glycans on proteins and acidic sphingolipids such as gangliosides and has important biological functions. The sialylation process is controlled by more than 20 different sialyltransferases, many of which exhibit overlapping functions. Thus, it is difficult to determine the overall biological function of sialylation by targeted deletion of individual sialyltransferases. To address this issue, we established a mouse line with the Slc35a1 gene flanked by loxP sites. Slc35a1 encodes the cytidine-5'-monophosphate (CMP)-sialic acid transporter that transports CMP-sialic acid from the cytoplasm into the Golgi apparatus for sialylation. Here we report our study regarding the role of sialylation on megakaryocytes and platelets using a mouse line with significantly reduced sialylation in megakaryocytes and platelets (Plt Slc35a1-

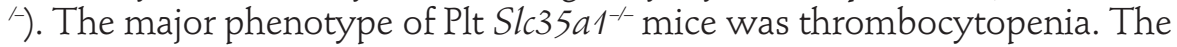

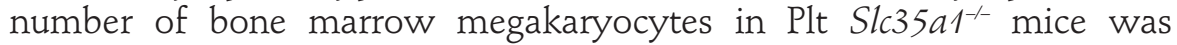
reduced, and megakaryocyte maturation was also impaired. In addition, an increased number of desialylated platelets was cleared by Küpffer cells in the liver of Plt Slc35a $1^{-1}$ mice. This study provides new insights into the role of sialylation in platelet homeostasis and the mechanisms of thrombocytopenia in diseases associated with platelet desialylation, such as immune thrombocytopenia and a rare congenital disorder of glycosylation (CDG), SLC35A1-CDG, which is caused by SLC35A1 mutations.

\section{Introduction}

Platelets are among the most abundant blood cells in circulation. In addition to their well-established roles in hemostasis and thrombosis, platelets are involved in a broad spectrum of other physiological and pathological processes such as vascular integrity, immunity, inflammation, and tumor metastasis. ${ }^{1.5}$ A stable number of platelets is of great importance to these functions. Platelet homeostasis is primarily regulated by the production and clearance of platelets. Platelet membrane receptors play a vital role in platelet hemostasis. Most of these proteins are glycoproteins (GP), including the GPIb-IX-V complex and GPIIb/IIIa complex. ${ }^{6}$ Major forms of glycosylation include $\mathrm{N}$-linked glycans ( $\mathrm{N}$-glycans) and mucin-type $\mathrm{O}$-linked glycans (O-glycans). Both $\mathrm{N}$ - and O-glycans are commonly "capped" by sialic acids, a process named sialylation. ${ }^{8,9}$ Sialylation plays essential biological roles, especially in cell-cell interactions. ${ }^{8}$ The significance of sialylation in other biological processes,
Ferrata Storti Foundation

Haematologica 2021

Volume 106(3):759-769

\section{Correspondence:}

LIJUN XIA

Lijun-xia@omrf.org

Received: May 7, 2019.

Accepted: March 19, 2020.

Pre-published: April 17, 2020.

https://doi.org/10.3324/haematol.2019.225987

(C)2021 Ferrata Storti Foundation

Material published in Haematologica is covered by copyright. All rights are reserved to the Ferrata Storti Foundation. Use of published material is allowed under the following terms and conditions:

https://creativecommons.org/licenses/by-nc/4.0/legalcode. Copies of published material are allowed for personal or internal use. Sharing published material for non-commercial purposes is subject to the following conditions:

https://creativecommons.org/licenses/by-nc/4.0/legalcode, sect. 3. Reproducing and sharing published material for commercial purposes is not allowed without permission in writing from the publisher. 
such as platelet homeostasis, has just begun to be appreciated.

$\alpha 2,3$-linked sialic acid is the main form of platelet sialylation and is commonly linked to the penultimate galactose (Gal) or $N$-acetylgalactosamine (GalNAc) on complex $N$-glycans and O-glycans. ' The sialylation level on platelets is reduced in conditions such as cold storage of platelets, sepsis, and a subset of immune thrombocytopenia. ${ }^{10,11}$ It has been reported that desialylated platelets express terminal galactose and are cleared by hepatocytes through interactions with hepatic asialoglycoprotein receptor. ${ }^{12-14}$ This mechanism is considered to regulate normal platelet homeostasis and contributes to thrombocytopenia under pathological conditions. Our recent study revealed that Küpffer cells, rather than hepatocytes, phagocytose desialylated or O-glycan-deficient platelets. Glycosylation is also important for megakaryocytopoiesis and platelet production. Mice with constitutive or inducible global loss of O-glycans exhibited thrombocytopenia due to defects in terminal megakaryocyte differentiation and platelet production, demonstrating that $O$ glycosylation is critical for thrombocytogenesis. ${ }^{15,16}$

Sialylation occurs in the trans-Golgi by transferring sialic acids from the donor substrate cytidine-5'-monophosphate-sialic acid (CMP-SA) to acceptor $N$ - and/or O-glycans (Figure 1A), ${ }^{8,9}$ There are de novo and salvage pathways of CMP-SA biosynthesis. In the de novo pathway, sialic acids are synthesized in the cytoplasm from the substrate uridine diphosphate (UDP)-N-acetylglucosamine through serial reactions catalyzed by the enzymes UDP-N-acetylglucosamine 2-epimerase, $\mathrm{N}$-acetylneuraminic acid synthase, and N-acetylneuraminate-9-phosphate-phosphatase. On the other hand, sialic acid can also be generated during desialylation of sialylated molecules. Sialic acids are attached to CMP catalyzed by CMP-SA synthase in the nucleus, and subsequently CMP-SA is transported into the Golgi lumen by the CMP-sialic acid transporter SLC35A1.

There are more than 20 known genes in mice and humans encoding sialyltransferases. $\alpha 2,3$-linked sialic acids on glycans of megakaryocytes and platelets are generated by six different $\alpha 2,3$-sialyltransferases (ST3Gal-I VI). Studies with mice lacking ST3Gal-I or ST3Gal-IV have shown the importance of sialylation in platelet biology, especially platelet clearance by hepatocytes and/or Küpffer cells. ${ }^{17}$ However, due to redundant expression of these sialyltransferases in platelets, such as ST3Gal-I, ST3Gal-IV, and ST6Gal-I, ${ }^{18,19}$ the overall function of sialylation in platelet homeostasis remains to be studied.

The CMP-SA transporter (CST) is encoded by the SLC35A1 gene (Figure 1A). Several patients with SLC35A1 mutations have been reported and are described as having

A

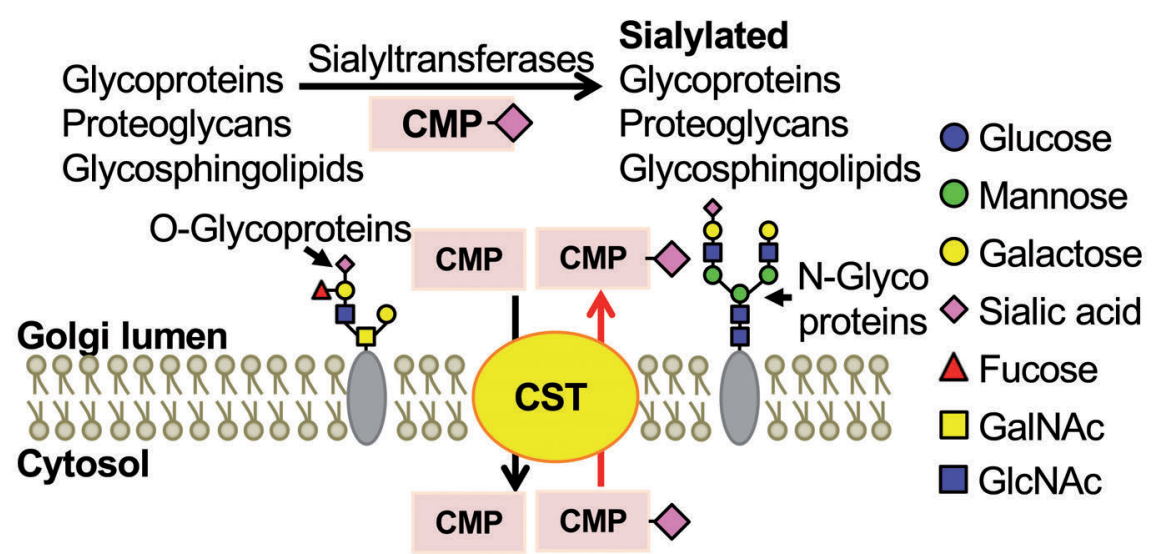

B

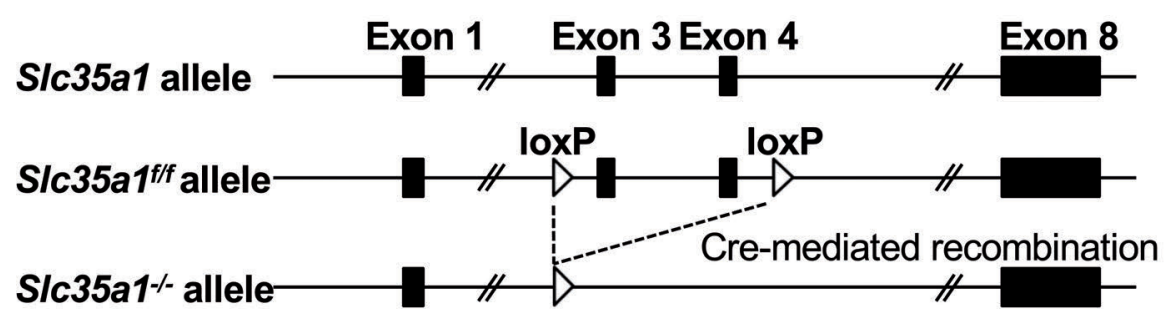

Figure 1. Generation of mice with megakaryocyte- and platelet-specific deletion of SIc35a1. (A) The function of S/c35a1-encoded CMP-sialic acid transporter (CST). (B) Targeting strategy for conditional deletion of the S/c35a1 gene. Diagram of wild-type (WT) (Slc35a1), loxP site-flanked $\left(\right.$ S/c35a $\left.1^{t / 7}\right)$, and null (S/c35a $\left.1^{-/}\right)$ alleles of S/c35a1. Arrowheads indicate the position of loxP. (C) Genotyping polymerase chain reaction of tail genomic DNA from offspring was used to identify PIt Slc35a $1^{-1}$ mice. Lane 3 represents the genotype of PIt Slc35a1 $1^{-/}$mice. (D) S/c35a1 gene expression in WT and Plt Slc35a $1^{-/}$mice was determined by quantitative reverse transcriptase polymerase chain reaction using RNA extracted from bone marrow megakaryocytes ( $n=3$ for

each group).

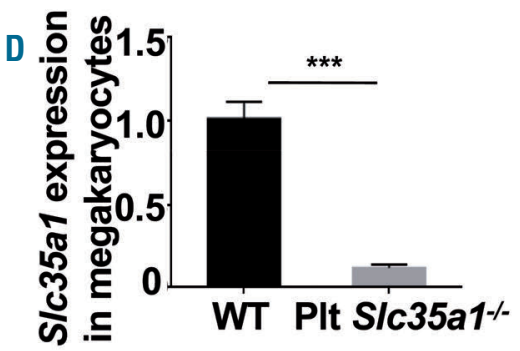


an SLC35A1-related congenital disorder of glycosylation (SLC35A1-CDG). Clinical manifestations in these rare SLC35A1-CDG patients are complex, including neutropenia, opportunistic infections, delayed psychomotor development, epilepsy, ataxia, microcephaly and choreiform..$^{20}$.

${ }^{23}$ However, a common manifestation in these patients is macrothrombocytopenia. Accelerated platelet clearance, but not impaired proplatelet production, is considered to contribute to the thrombocytopenia based on in vitro analyses. ${ }^{23}$

CST is a critical transporter to exchange CMP-sialic acid for CMP across the Golgi membrane, which is essential for sialylation, using an antiport mechanism (Figure 1A). ${ }^{24}$ So far, no mouse model with deficiency of CMP-SA is available to study this key process. For this reason, we generated genetically-targeted mice with exons 3 and 4 of Slc35at being flanked by loxP sites (Slc35atrif). In this study, we established a mouse model lacking Slc35a1 specifically in megakaryocytes and platelets (Plt Slc35a1 ${ }^{-1}$ ) to determine whether desialylation affects platelet homeostasis. Plt Slc35a1 $1^{-1}$ mice exhibited thrombocytopenia with impaired megakaryocyte maturation and increased platelet clearance. This study provides new insights into the role of sialylation in platelet production and clearance.

\section{Methods}

Based on our published methods, ${ }^{7,25}$ we first generated conditional mice with the Slc35a1 gene flanked by loxP sites (Slc35atff).

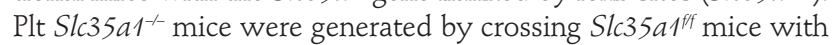
a transgenic mouse line expressing Cre recombinase under the control of the mouse $P f 4$ promoter ( $P f 4$ Cre mice, The Jackson Laboratory, \#008535) (Figure 1B). Mice were bred and maintained in specific pathogen-free conditions in the Laboratory Animal Experimental Center at Soochow University and Oklahoma Medical Research Foundation. Mouse studies were approved by the Animal Use Committee of the First Affiliated Hospital of Soochow University and Oklahoma Medical Research Foundation.

\section{Platelet preparation and analysis}

Platelets were isolated and prepared based on our previous publications. ${ }^{3,7,26}$ In brief, whole blood was obtained from the inferior vena cava and collected into test-tubes containing sodium citrate as an anticoagulant, diluted 1:1 with Tyrode buffer, and centrifuged at $100 \mathrm{~g}$ for $10 \mathrm{~min}$ at room temperature. The supernatant was collected and centrifuged at $800 \mathrm{~g}$ for $8 \mathrm{~min}$ to obtain the platelet pellet, followed by two subsequent washes with Tyrode buffer. Details are provided in the Online Supplementary Data.

\section{Flow cytometry}

Details of flow cytometry analysis of platelet sialylation profile, reticulated platelets, and glycoprotein expression on platelet surfaces are provided in the Online Supplementary Data.

\section{Analysis of bone marrow megakaryocytes}

Purification of megakaryocytes from the bone marrow, megakaryocyte counts of bone marrow paraffin-embedded sections, proplatelet formation assay, and assay of colony-forming unit-megakaryocyte are detailed in the Online Supplementary Data.

\section{Western blot analysis}

Freshly isolated megakaryocytes or platelets were washed with phosphate-buffered saline, and the pellet was re-suspended in cell lysis buffer (Cell Signaling Technology) containing protease inhibitor (1:100 dilution, Cell Signaling Technology). Further details are given in the Online Supplementary Data.

\section{Microscopic analysis}

Blood smears were fixed with methanol for $10 \mathrm{~min}$ at room temperature followed by Wright-Giemsa staining. For immunofluorescence microscopy, livers were obtained from mice after perfusion. OCT-embedded livers were cut into $8 \mu \mathrm{m}$ sections and stained with anti-F4/80 (Abcam) and anti-CD41 antibodies for Küpffer cells and platelets, respectively. After mounting, immunostaining was detected using an inverted confocal fluorescence microscope (TCSSP8, Leica). For transmission electron microscopy, bone marrow was fixed with $2.5 \%$ glutaraldehyde and embedded in Epon as described previously. ${ }^{27}$ Further details are provided in the Online Supplementary Data.

\section{Platelet glycan analysis}

Glycan structure was analyzed based on our published methods.? In brief, platelets isolated from wild-type (WT) or Plt

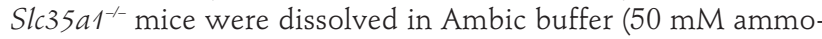
nium bicarbonate). Samples were heated for $5 \mathrm{~min}$ at $100^{\circ} \mathrm{C}$ for denaturation and then digested with trypsin $\left(37^{\circ} \mathrm{C}\right.$, overnight). After tryptic digestion, the $N$-glycans were released using PNGase F (New England BioLabs) and the $N$-linked glycan fraction was eluted through a C18 reversed phase cartridge. Subsequently, $O$-glycans were released by $\beta$-elimination. Both $N$-glycans and $O$-glycans were permethylated and analyzed by matrix-assisted laser-desorption time-of-flight (MALDI-TOF) tandem mass spectrometry (MS/MS) (Online Supplementary Figure S3)

\section{Statistics}

The unpaired Student t-test was used to determine $P$ values as indicated in the figures.

\section{Results}

\section{Generation of mice with megakaryocyte- and} platelet-specific deletion of SIc35a1

To generate the Slc35a1ff mice, exons 3 and 4 of the Slc35al gene were flanked with loxP sites (Figure 1B). Floxed Slc35a1 $1^{f /+}$ embryonic stem cells were injected into blastocysts of C57BL/6J mice to obtain chimeric mice. The derived mice were mated with C57BL/6J mice to achieve

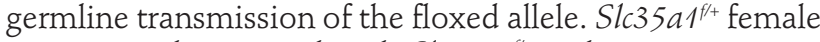
mice were then mated with Slc35a $1^{\text {ft }}$ male mice to gener-

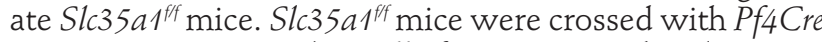

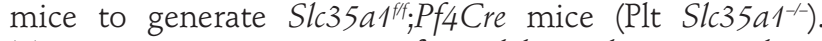
Mouse genotypes were confirmed by polymerase chain reaction (PCR) by using tail genomic DNA to amplify a 273-bp product and a 343-bp product from the WT allele and Slc35a1 floxed allele, respectively (Figure 1C). Slc35a1 expression in bone marrow megakaryocytes was abolished in Plt Slc35a1 1/- $^{-/}$mice as determined by quantitative reverse transcription PCR (Figure 1D).

To further confirm the Slc35a1 deletion in Slc35a1-1platelets, we amplified the major coding exons 3 to 6 of Slc35a1 by reverse transcription PCR. We detected a PCR amplicon of the expected size in cDNA from WT but not from Slc35a1 $1^{-/-}$platelets, indicating deletion of functional

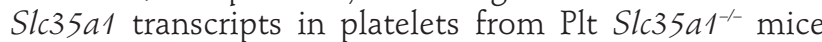
(Online Supplementary Figure S1). 
SIc35a1 ${ }^{-/}$platelets and megakaryocytes are deficient in sialylation

The glycosylation profiles of WT and S1c35a1-1 platelets and megakaryocytes were analyzed by flow cytometry and confocal microscopy based on lectin staining. For the lectin-based flow cytometry, we used biotinylated lectins, which enabled us to use PE-streptavidin only as a negative control. Neuraminidase-treated samples were used as a positive control (Figure 2A, Online Supplementary Figure S2). Binding of SNA (specific for $\alpha 2,6$-sialic acid) was not
A
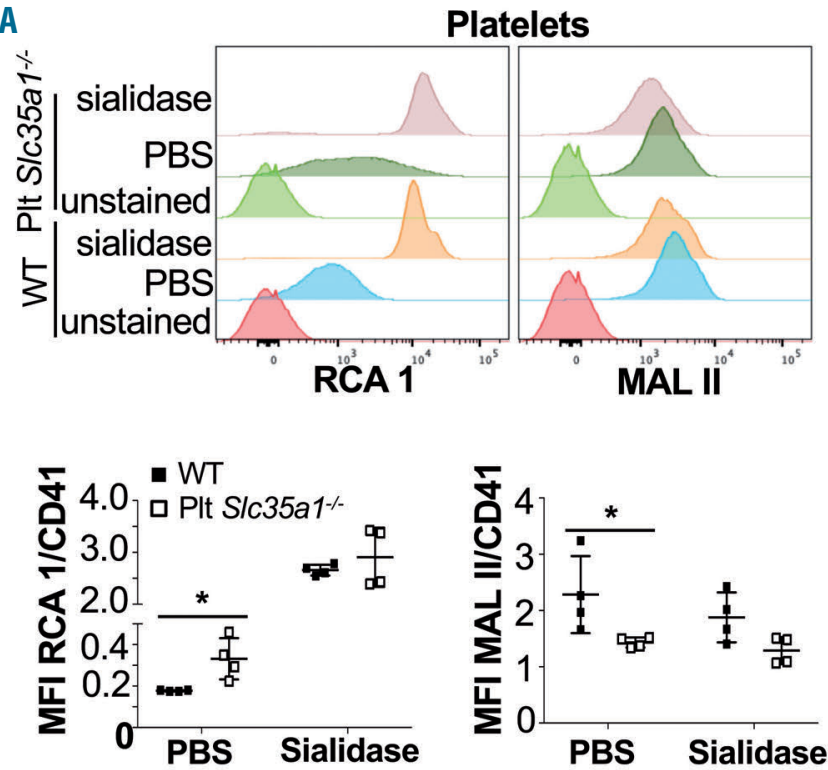

B
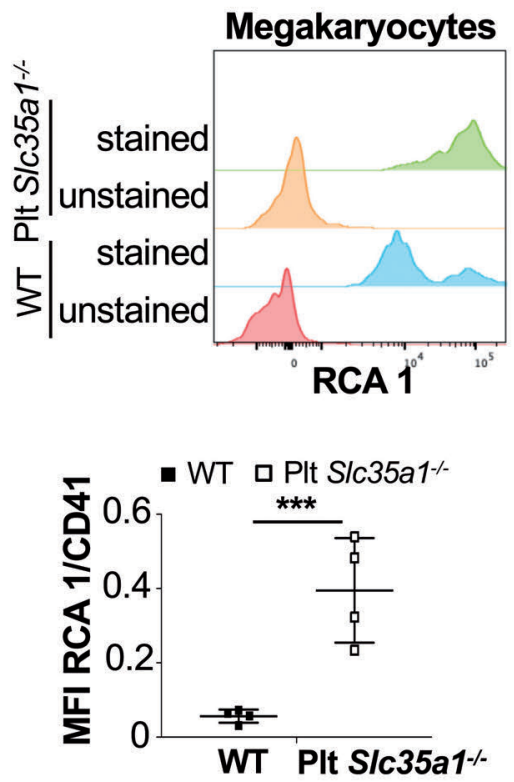

C
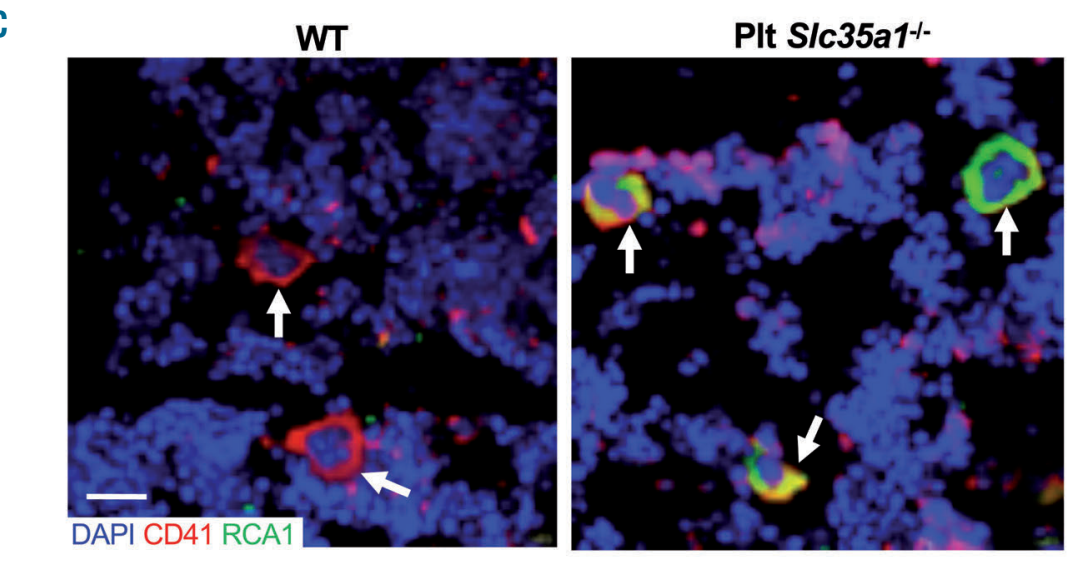

D

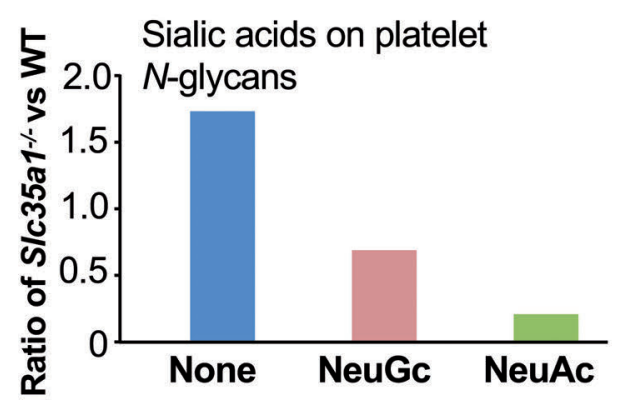

E

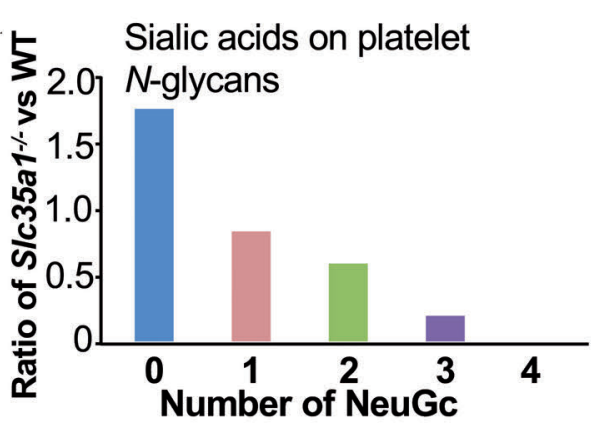

Figure 2. S/c35a1 ${ }^{-/}$platelets and megakaryocytes have reduced sialylation. (A) Top, a representative histogram of flow cytometry analysis of wild-type (WT) and SIc35a $1^{-/-}$platelets stained with biotinylated RCA 1 (specific for non-reducing terminal $\beta$-galactose), or biotinylated MAL II (specific for $\alpha 2,3-$ linked sialic acid), and FITC-labeled anti-CD41 antibody. Sialidase-treated platelets were used as a positive control. Platelets treated with phosphate-buffered saline (PBS) were used as a negative control. Unstained, incubation with PE-streptavidin only as a negative control; Bottom, mean fluorescence intensity ratios of RCA 1 versus CD41 or MAL II versus CD41 ( $n=4)$. (B) A representative histogram of flow cytometry analysis as shown in (A) on primary bone marrow megakaryocytes ( $n=4)$. (C) Representative confocal microscopic images of WT $(n=3)$ and SIc35a $1^{--}(n=3)$ bone marrow megakaryocytes. Arrows, megakaryocytes. Scale bar, $10 \mu \mathrm{m}$. (D and E) Ratio of molecular species of sialic acids between WT or S/c35a1 1 platelets. NeuGc, a major isoform of sialic acids in mice. NeuAc, a minor isoform of sialic acids in mice. The numbers $0-4$ in (E) indicate the number of NeuGc on complex N-glycans. $* P<0.05 ; * * * P<0.001$. 
observed on either WT or Slc35at- platelets (data not shown). RCA 1 (specific for terminal $\beta$-galactose) binding was increased in Slc35a 1 $^{-1}$ platelets compared to that in WT platelets (Figure 2A). In contrast, MAL II (specific for terminal $\alpha 2,3$-sialic acid) binding was statistically decreased in Slc35a1-- platelets when compared to its biding in WT platelets (Figure 2A). To determine whether platelet size affected the lectin measurement, we costained lectins with anti-CD41 antibody and calculated ratios of their mean fluorescent intensity (MFI). The MFI of RCA 1/CD41 increased significantly while the MFI of MAL II/CD41 decreased significantly in Slc35a $1^{-1}$ platelets compared to the intensities in WT platelets (Figure 2A). All Slc35a $a 1^{-1}$ megakaryocytes were positive for RCA 1 staining by both flow cytometry and confocal microscopy analysis (Figure $2 \mathrm{~B}$ and $\mathrm{C}$ ). However, for unknown reasons, we were unsuccessful in acquiring reproducible MAL II staining results either by flow cytometric analysis or immunostaining of megakaryocytes. These results demonstrated that disruption of Slc35a1 results in a significant reduction of sialylation in Slc35a1 $1^{-1-}$ megakaryocytes and platelets.

To complement these lectin-based analyses, we released, purified, and permethylated $\mathrm{N}$ - and $\mathrm{O}$-linked glycans from each platelet sample, then performed MALDI-TOF mass spectrometry to determine the platelet sialylation pattern, followed by nanospray ionizationMS/MS to confirm several structures by collision-induced dissociation (Figure 2D and E; Online Supplementary Figures $\mathrm{S} 3$ and S4). For $\mathrm{N}$-glycosylation, there was a significant increase of un-sialylation (from $28.6 \%$ to $50.1 \%$ ) in Slc35a $1^{-1}$ platelets, whereas $N$-glycolylneuraminic acid (NeuGc), a major isoform of sialic acids in non-human mammals, exhibited a decreased trend (from $71.4 \%$ to $49.9 \%$ ). Moreover, $\mathrm{NeuGc}$ isoform analysis showed that one of the NeuGc isoforms was undetectable in Slc35at $1^{-1-}$ platelets (Online Supplementary Figure S4A). Glycan structure analysis indicated that $O$-glycans such as sialylated (NeuGc) core 1 structures at $\mathrm{m} / \mathrm{z} 669$ decreased significantly in Slc35 $a^{--}$platelets (Online Supplementary Figure S4B, Online Supplementary Table S1).

We noticed that residual sialylation was detected by the high resolution MALDI-TOF-MS/MS analysis in Slc35a 1- $^{-1}$ platelets. As Plt Slc35a1 ${ }^{--}$mice have normal sialylation in cells including hematopoietic cells other than megakyocytes/platelets, we hypothesized that residual sialylation in Slc35a1 ${ }^{-1}$ platelets is caused by exogenous plasma sialylated molecules, such as fibrinogen and IgG which are abundant in the plasma and known to be sialylated, internalized by circulating platelets. ${ }^{28.31}$ In addition, exogenous $\operatorname{IgG}$ is also commonly found on the surface of platelets (PAIgG) ${ }^{30,31}$ This can be reflected in the fact that the relative ratios of singly to doubly-sialylated glycoforms remained consistent to each other in the WT and Slc35a1-

platelets, rather than the number of doubly-sialylated glycoforms decreasing in addition to the singly-sialylated and non-sialylated species increasing in incomplete Slc35a $a 1^{-1}$ platelets. To test the hypothesis that exogenous sialylated plasma proteins contributed to the residual sialic acids in the Slc35at- platelets, we immunoprecipitated IgG from platelet lysates using protein $A / G$ beads, and then blotted the immunoprecipitated IgG with MALII lectin that binds to $\alpha 2,3$-sialic acids. Our results indicated that both WT and Slc35a1-- platelets contained MALIIpositive IgG (Online Supplementary Figure S5). As megakaryocytes and platelets do not synthesize IgG, this result indicates that Slc35a $1^{-1-}$ platelets contain exogenous sialylated proteins, which supports our hypothesis. On the basis of all these findings, we concluded that Slc35a1- megakaryocytes and platelets have significantly reduced sialylation (Figure 2; Online Supplementary Figures S4 and S5; Online Supplementary Table S1), thus being an appropriate model to study the effect of sialylation on endogenous sialylated molecules in megakaryogenesis and platelet homeostasis.

To determine whether abnormal sialic acid accumulation existed or not, we checked free sialic acid levels in serum and found that the sialic acid levels were identical

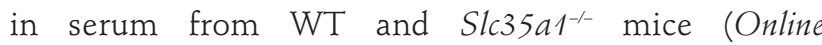
Supplementary Figure S6).

\section{PIt SIc35a1 ${ }^{-/}$mice exhibit macrothrombocytopenia}

The peripheral platelet count of Plt Slc35at-- mice was significantly lower than that of WT mice, and the mean platelet volume of Slc35a1-1- platelets was significantly larger than that of WT platelets (Figure 3A and Online Supplementary Figure S7A). Giemsa staining of blood smears also confirmed fewer platelets in Plt Slc35a $1^{-1-}$ mice (Figure 3B). Tail bleeding time analysis showed no significant difference between WT and Plt Slc35a 1-- $^{-1}$ mice (Online Supplementary Figure S7B). Since thrombopoietin regulates megakaryopoiesis and platelet formation, we measured plasma thrombopoietin level and found that it was significantly increased in Plt Slc35a1 $1^{-/}$mice (Figure 3C). However, although the percentage of reticulated platelets was higher, the absolute count of reticulated platelets in Plt Slc35a $1^{-1-}$ mice was lower than that in WT mice (Figure $3 \mathrm{D}$ and $\mathrm{E})$. These results suggest that platelet production was impaired in Plt Slc35a $a^{-1-}$ mice.

There was also no difference in leukocyte count and hemoglobin content in peripheral blood between WT and Plt Slc35a $1^{-1-}$ mice (Online Supplementary Figure S7C and D). Histology showed no abnormality of major organs (liver, spleen, heart and kidney) in Plt Slc35a $a^{--}$mice (Online Supplementary Figure S8).

\section{SIc35a1-1- platelets express lower levels of GPIb $\alpha$}

Platelet GPIb $\alpha$ (CD42b) and integrin aIIb33 (CD41, CD61) are heavily glycosylated. To examine whether Slc35a1 deficiency impaired the surface levels of platelet glycoproteins, we measured GPIb $\alpha$ and integrin $\alpha \operatorname{IIb} \beta 3$ on WT and Slc35a $a^{-1}$ platelets by flow cytometry. Our results showed that the level of GPIb $\alpha$ was decreased on Slc35 $a 1^{-1-}$ platelets (Figure 4A). However, no difference in integrin $\alpha I I b \beta 3$ level was observed between WT and Slc35a $a^{-1 /}$ platelets (Figure 4A). Likewise, there was no significant differences in GPIb $\alpha$ and integrin $\alpha \mathrm{IIb} \beta 3$ levels between WT and Slc35a $1^{-1-}$ megakaryocytes (Figure 4B). Western blot analysis indicated reduced total GPIb $\alpha$ content in Slc35a1- platelets (Online Supplementary Figure S9), suggesting that GPIba shedding, but not internalization, is likely the cause of the reduced level of GPIba on Slc35a $1^{-/}$platelets..$^{33,34}$

\section{Deletion of SIc35a1 results in impaired megakaryocyte differentiation and maturation}

Platelets are primarily produced by megakaryocytes in the bone marrow. Plt Slc35a $a^{-1}$ mice exhibited severe thrombocytopenia, raising the question of whether platelet production was affected by the absence of Slc35a1. 
A

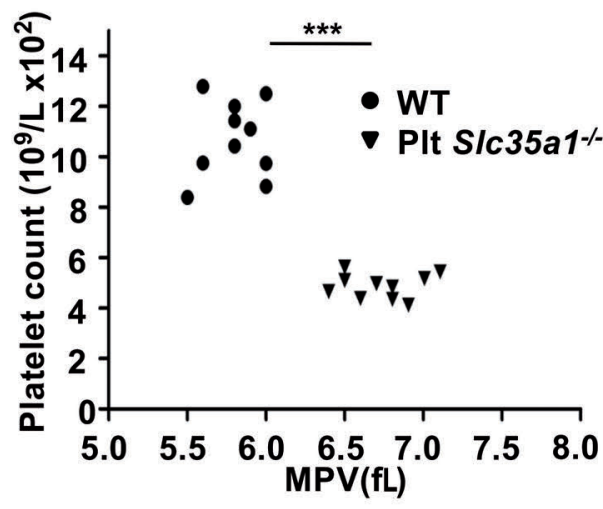

B
C

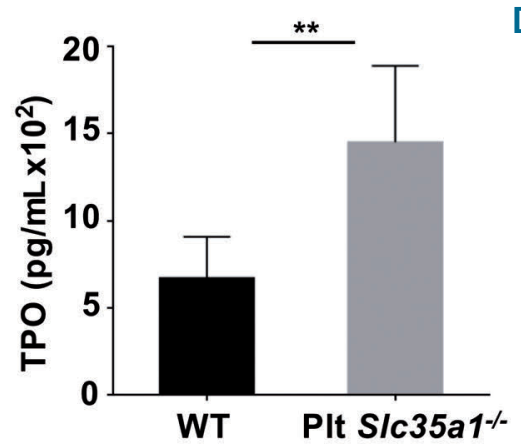

D

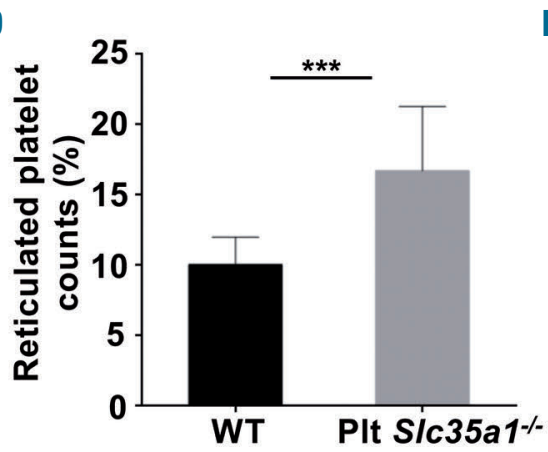

PIt SIc35a1\%

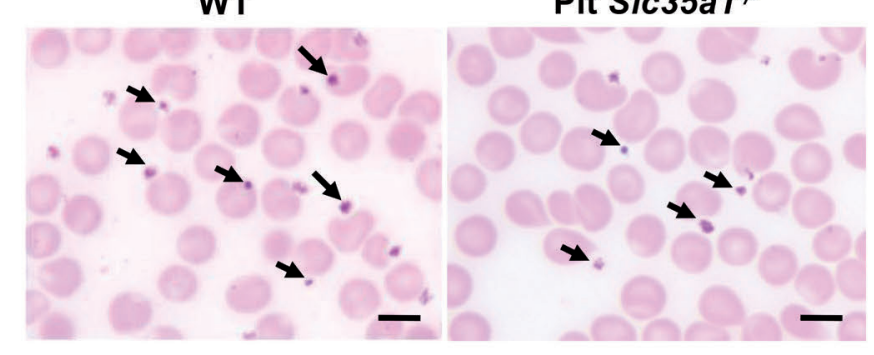

Figure 3. PIt S/c35a1\% mice exhibit thrombocytopenia. (A) Peripheral blood platelet counts and mean platelet volume in wild-type (WT) ( $\mathrm{n}=10$ ) and PIt S/c35a1 $(n=10)$ mice. Each circle or triangle represents one mouse. $* * * P<0.001$. (B) Wright-Giemsa staining of peripheral blood smears from WT ( $n=3)$ and PIt S/c35a1 $(n=3)$ mice. Platelets are indicated by arrows. Scale bar, $16 \mu \mathrm{m}$. (C) Plasma thrombopoietin levels of WT $(n=5)$ and PIt S/c35a1 ${ }^{--}(n=5)$ mice measured by enzymelinked immunosorbent assay. Data are means \pm standard deviation (SD). $* * P<0.01$. (D) Flow cytometry analysis of the percentage of reticulated platelets stained by anti-CD41 antibody and thiazole orange (TO) in WT $(n=12)$ and PIt S/c35a $1^{-/}(n=12)$ mice. Data are means \pm SD. $* * * P<0.001$. (E) Reticulated platelet counts of WT $(n=9)$ and PIt SIc35a1 ${ }^{--}(n=9)$ mice after staining with anti-CD41 antibody and thiazole orange. Data are means \pm SD. $* P<0.05$. MPV: mean platelet volume; TPO: thrombopoietin.

A

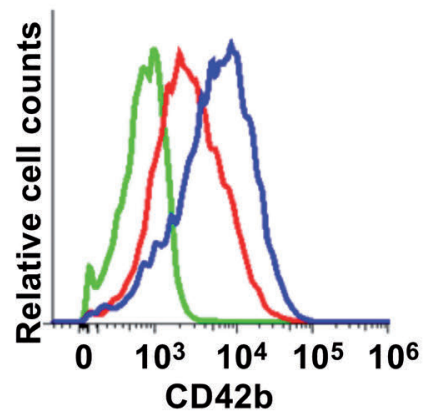

B

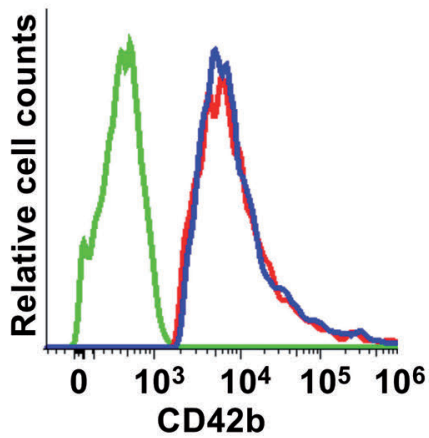

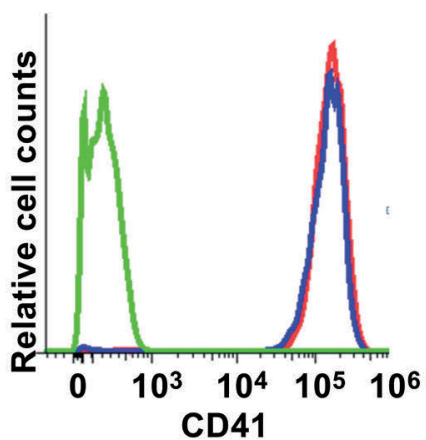
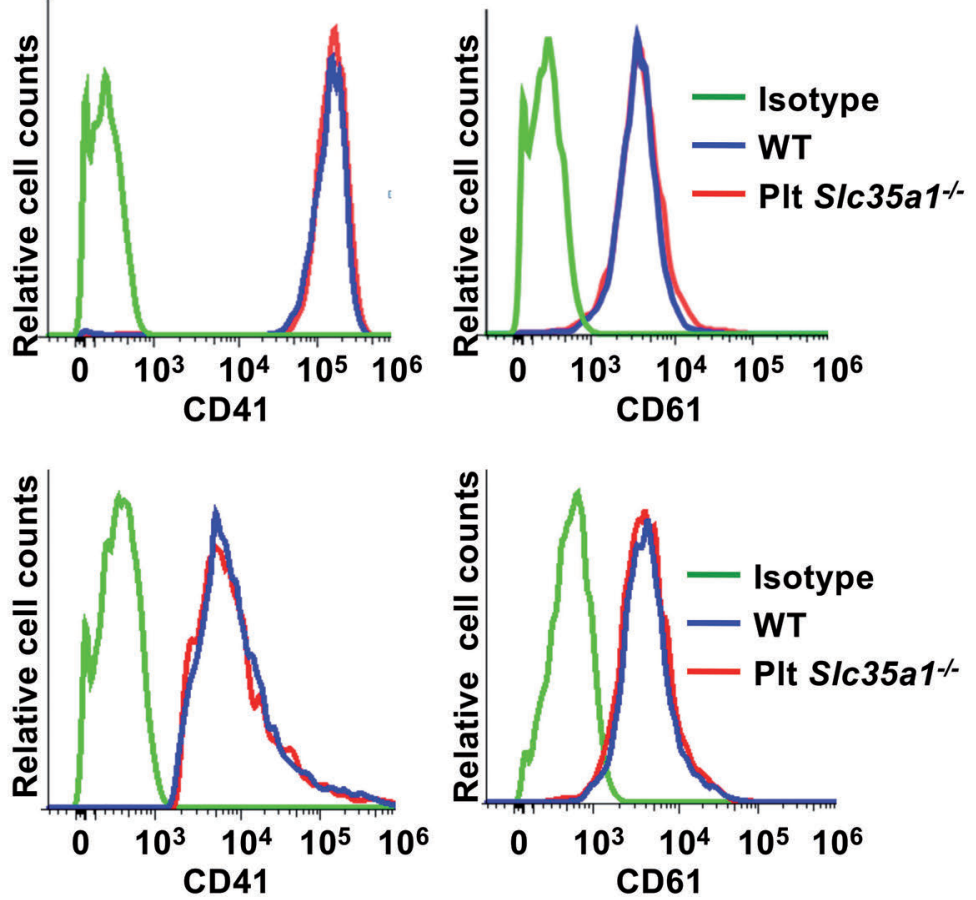

Figure 4. SIc35a1 ${ }^{--}$platelets express low levels of GPIba. (A) Wild-type (WT) and S/c35a1 ${ }^{-/}$platelets isolated from peripheral blood were stained with antibodies to CD42b, CD41 and CD61, and then analyzed by flow cytometry for expression of GPIba, GPIlb-Illa complex ( $\mathrm{n}=4$ for WT and S/c35a1 ${ }^{-1-}$ mice, respectively). (B) Megakaryocytes were isolated from WT or S/c35a1 ${ }^{-/}$bone marrow, and were then stained with antibodies to CD42b, CD41 and CD61. The stained samples were analyzed by flow cytometry for expression of GPIb $\alpha$ and GPIIb-IIla complex ( $n=3$ for WT and S/c35a $1^{- \text {mice, }}$, respectively). 
Hematoxylin \& eosin-stained, paraffin-embedded sections

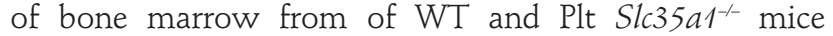
showed significantly decreased numbers of megakaryocytes (Figure 5A). A colony-forming unit-megakaryocyte assay was performed to determine the proliferation and differentiation of megakaryocytes, and we found that the proliferation potential of megakaryocytes from Plt Slc35a1 mice was decreased (Figure 5B). Giemsa-stained bone marrow smears indicated that megakaryocyte development and maturation were impaired in Plt Slc35a1 ${ }^{-1}$ mice relative to these processes in the WT mice (Figure 5C). At the ultrastructural level, the WT megakaryocytes exhibited a distinct granular zone containing a well-developed demarcation membrane system (Figure 5D, top). In con-

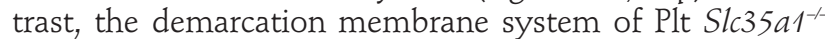
megakaryocytes was disorganized with fewer well-delimited platelet territories (Figure 5D, bottom).

To determine which developmental stages of megakaryocytes are affected by the loss of Slc35a1, we used multicolor flow cytometry to analyze isolated bone marrow cells (Online Supplementary Figure S10). Flow cytometry detected no difference in the percentage of megakaryocyte/erythroid progenitors and megakaryocyte progenitors between WT and Plt Slc35a1 $1^{-/-}$mice (Figure 6A). ${ }^{35,36}$ Megakaryocyte differentiation correlates with increased DNA content, so we examined DNA ploidy of bone marrow megakaryocytes from WT and Plt Slc35a1 ${ }^{-1}$ mice by flow cytometry (Online Supplementary Figure S11). Ploidy analysis using Hoechst33342 staining on bone marrow cells showed a significantly higher percentage of megakaryocytes at the early $2 \mathrm{~N}$ stage in Slc35a1 ${ }^{-1-}$ mice than in WT mice (Figure 6B). To determine whether

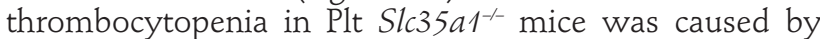
defects in the production of proplatelets from megakaryocytes, we performed a proplatelet formation assay. CD41 megakaryocytes with cytoplasmic pseudopods longer than the diameter of cytoplasm were defined as proplatelet-forming megakaryocytes (Figure 6C). Fibrinogen-
A

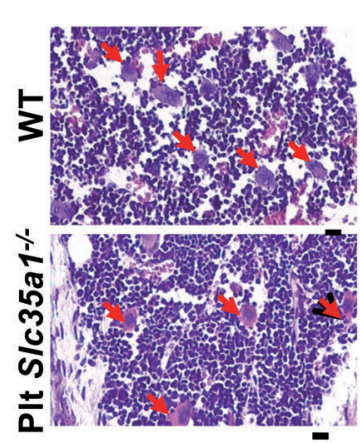

B

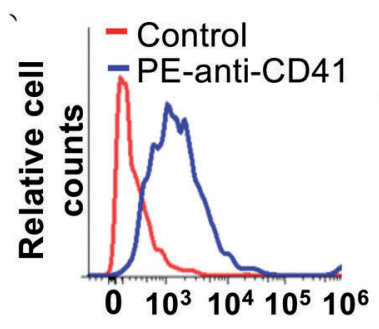

C

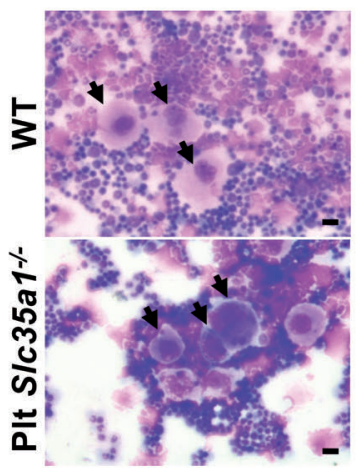

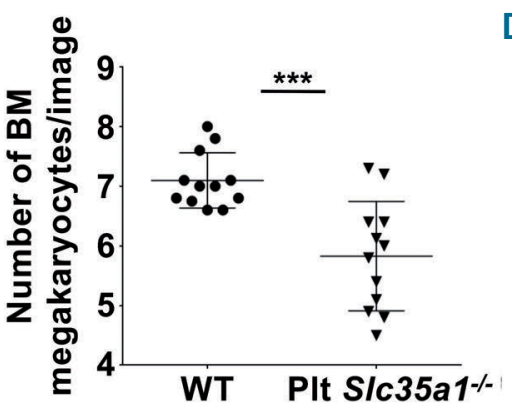

D

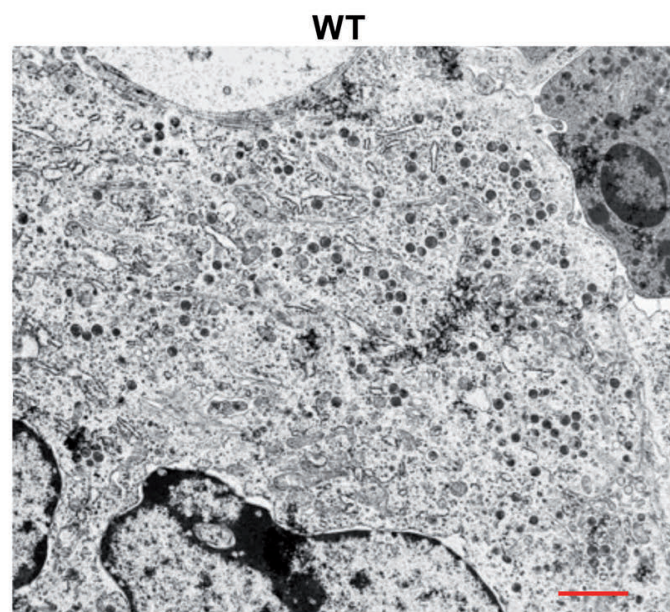

PIt SIc35a1-

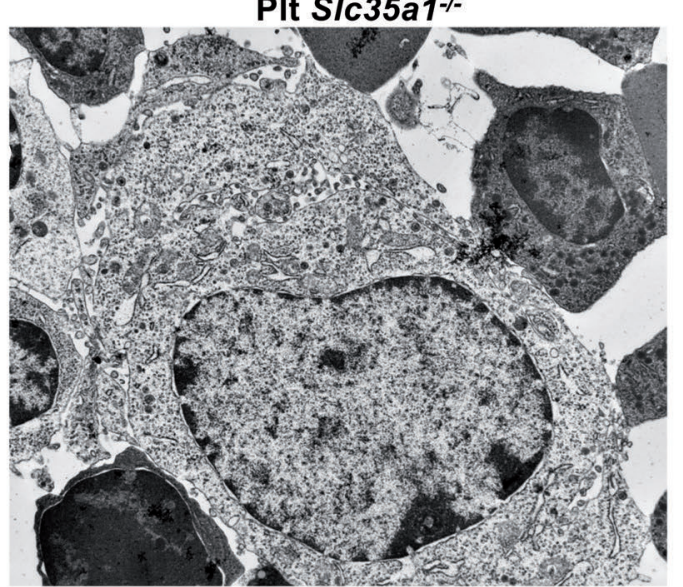

Figure 5. PIt SIc35a1 $/-$ mice exhibit decreased megakaryocyte counts and defective megakaryocytopoiesis in bone marrow. (A) Representative images of hematoxylin \& eosin-stained wild-type (WT) and PIt S/c35a1 1 - bone marrow (BM) paraffin-embedded sections (left). Analysis of BM megakaryocyte counts per image of WT and PIt SIc35a1 $1^{--}$mice (right). Three images/mouse of 12 mice per genotype. Arrows mark megakaryocytes. Data are means \pm standard deviation (SD). Scale bar, $20 \mu \mathrm{m}$. (B) The colony-forming unit-megakaryocyte assay. Colonies were enumerated using an inverted microscope (right). Colonies were also stained with antiCD41 antibody and detected by flow cytometry (left). Data are means \pm SD. WT, $n=10$; PIt S/c35a1\%, $n=6$. (C) Analysis of different stages of megakaryocytes using BM smears stained with Giemsa from WT $(n=6)$ and PIt S/c35a $1^{--}(n=6)$ mice. Data are means \pm SD. Scale bar, $20 \mu \mathrm{m}$. (D). Representative transmission electron microscopy micrographs of BM megakaryocytes ( $\mathrm{n}=3$ for WT and SIc35a1 $1^{--}$mice, respectively). Scale bar, $2 \mu \mathrm{m}$. $* * * P<0.001$. 
A

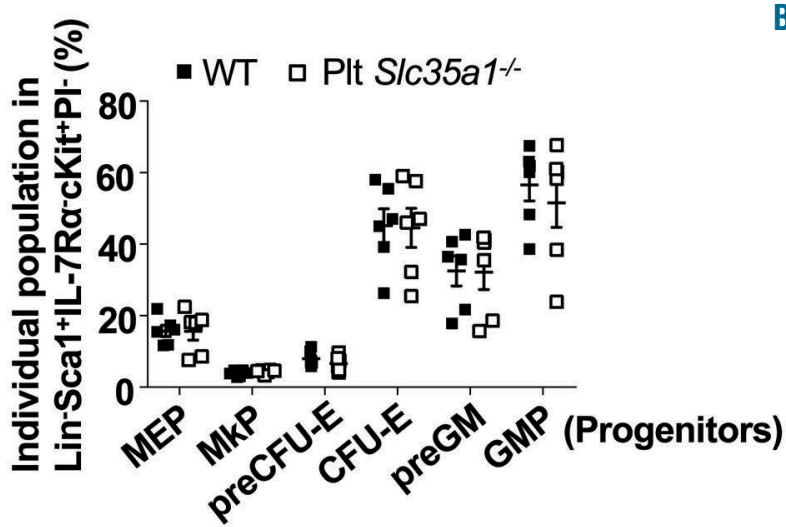

B

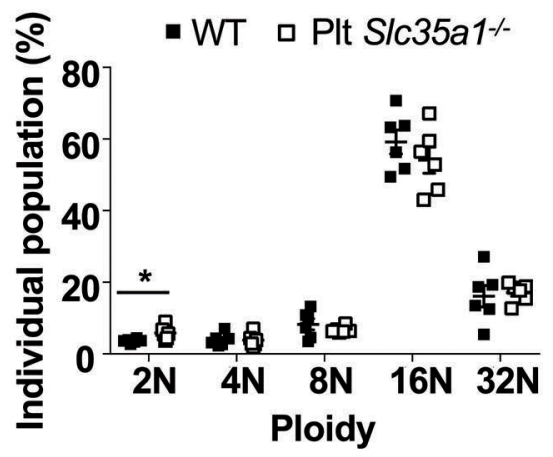

C

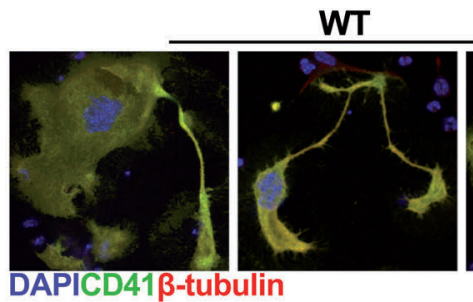

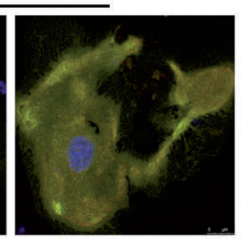

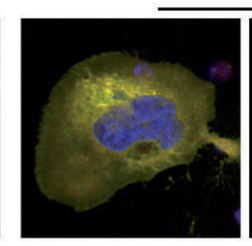

PIt SIc35a1-1-

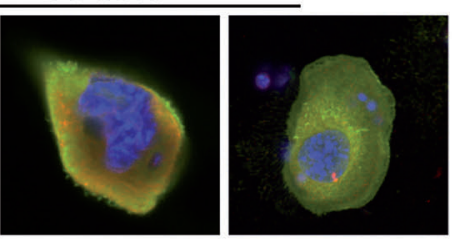

\begin{tabular}{c|c|c}
\hline \multirow{2}{*}{$\begin{array}{c}\text { Total number } \\
\text { of CD41 }\end{array}{ }^{+}$cells } & \multicolumn{2}{|c}{ Number of proplatelet-forming megakaryocytes (\%) } \\
\cline { 2 - 3 } & WT & PIt S/c35a1 $1^{-}$ \\
\hline $40 \times 4$ & $98(61.25 \pm 5.82 \%)$ & $52(32.5 \pm 2.28 \%)^{\star *}$ \\
\hline
\end{tabular}

** $P<0.01$

Figure 6. SIc35a1 $1^{--}$megakaryocytes exhibit defective maturation. (A) Quantification of individual progenitors in bone marrow. Megakaryocyte progenitors were defined as Lin CD127 Sca1 $\mathrm{CKit}^{+} \mathrm{CD} 150^{+} \mathrm{CD} 41^{+}$bone marrow cells. Megakaryocyte erythroid progenitors were defined as Lin CD127-Sca1 KKit $^{+} \mathrm{CD} 150^{+} \mathrm{CD} 41 \mathrm{CD} 16 / 32$ CD105 bone marrow cells. Data are mean \pm standard error of mean (SEM). $* * P<0.01, n=8$ mice/genotype. (B) Polyploidy quantification of bone marrow megakaryocytes. Bone marrow megakaryocytes stained with PerCP-Cy5.5-CD41 and Hoechst33342 were subject to ploidy analysis by flow cytometry. Data are means \pm SEM. $\mathrm{n}=8$ mice/genotype. (C) Representative images of proplatelet-forming megakaryocytes in CD $41^{+}$cells. CD $41^{+}$megakaryocytes with cytoplasmic pseudopodia longer than the diameter of cytoplasm were defined as proplatelet-forming megakaryocytes. Bar, $10 \mu \mathrm{m}$. Quantification of proplatelet-forming megakaryocytes based on staining with anti-CD41 and anti- $\beta$-tubulin antibodies (table below). Data are mean \pm standard deviation. $n=8$ mice/genotype. $* * P<0.01$. MEP, megakaryocyte erythroid progenitor; MkP, megakaryocyte progenitor; preCFU-E, pre-colony forming unit-erythroid; CFU-E, colony forming unit-erythroid; preGM, pre-granulocytic monocytic progenitor; GMP, granulocytic-monocytic progenitor.

induced proplatelet formation identified by immunostaining with anti- $\beta$-tubulin and anti-CD41 antibodies showed that megakaryocytes in Plt Slc35a1-- mice displayed a reduced percentage of proplatelet-forming megakaryocytes when compared to the percentage in WT mice (Figure 6C). In addition, proplatelets from Plt Slc35a1-1 megakaryocytes were shorter and less branched compared to those from WT megakaryocytes.

The interaction of thrombopoietin and its receptor $\mathrm{Mpl}$ is critical for platelet production. $N$-glycosylation has been reported to regulate Mpl stability and function. ${ }^{37}$ In our study, western blot analysis showed that Mpl was decreased in Slc35a1 $1^{-1}$ bone marrow megakaryocytes (Figure 7A and B). Megakaryocyte-specific $M p l$ transcripts of WT and Plt Slc35a1 ${ }^{-1-}$ mice were analyzed by quantitative reverse transcription PCR, but no significant difference was detected, indicating that the reduction in $\mathrm{Mpl}$ may be due to decreased stability (Figure 7C).

\section{Desialylated platelets of PIt SIc35a1 ${ }^{-/}$mice are}

cleared by Küpffer cells in the liver

Glycan modifications regulate platelet clearance. ${ }^{7,13}$ To confirm whether this mechanism contributes to thrombocytopenia in Plt Slc35 $\mathrm{a1}^{--}$mice, we first analyzed liver and spleen by immunofluorescence staining. Spleen is the major organ for platelet clearance, but there was no difference in the numbers of platelets in spleens from WT and Plt Slc35a1-- mice (data not shown). Our previous study indicated that Küpffer cells in the liver are critical for the clearance of desialylated platelets.' We therefore performed confocal imaging analysis of liver sections from WT and Plt Slc35a1-- mice after staining with anti-F4/80 and anti-CD41 antibodies. The results showed that CD41 Slc35a1- platelets are primarily in contact with $\mathrm{F} 4 / 80^{+}$ Küpffer cells (Figure 8A and B). These data indicate that desialylated Slc35a1 $1^{-1}$ platelets are mainly cleared by Küpffer cells in the liver.

\section{Discussion}

As a common terminal glycosyl group, sialic acid can be attached to non-reducing terminal galactose $(\mathrm{Gal})$ residue via $\alpha 2,3-$, or $\alpha 2,6$-linkage, to GalNAc via $\alpha 2,6$-linkage, and to sialic acid via $\alpha 2,8$-linkage. ${ }^{8,9}$ The biosynthesis of these diversified forms of sialylation is controlled by more than 20 different sialyltransferases. ${ }^{11,19,38}$ Even though these enzymes are differentially expressed in different tissues to 


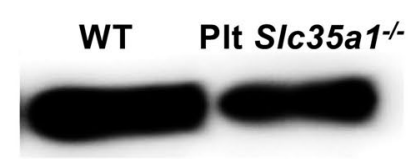

IB: anti-MpI

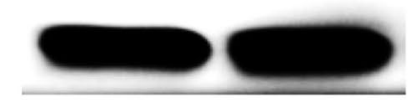

IB: anti- $\beta$-actin
B

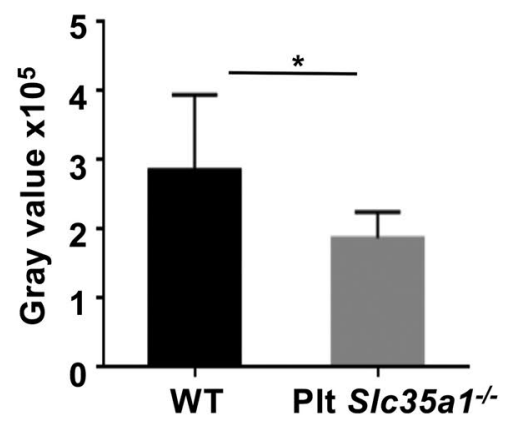

C

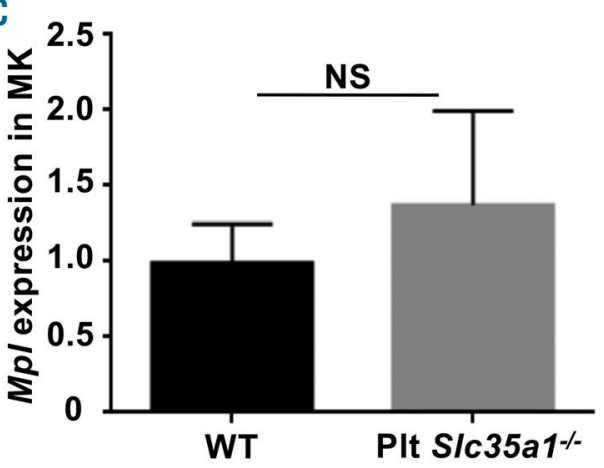

Figure 7. Mpl expression in SIc35a1\% bone marrow megakaryocytes is decreased. (A) Mpl expression on wild-type (WT) ( $=8$ mice) and S/c35a1\% ( $=7$ mice) megakaryocytes was analyzed with anti-Mpl antibody by western blot. $\beta$-actin was used as a loading control. (B) Quantification of Mpl expression in WT (n=8) and PIt S/c35a1\% megakaryocytes $(n=7)$ by densitometry of the western blot data. Data are means \pm standard deviation (SD). * $P<0.05$. (C) Quantification of $M p /$ transcripts in WT $(n=6)$ and PIt SIc35a1 bone marrow megakaryocytes $(n=6)$. Data are means \pm SD. IB: immunoblot; NS not statistically significant.

A

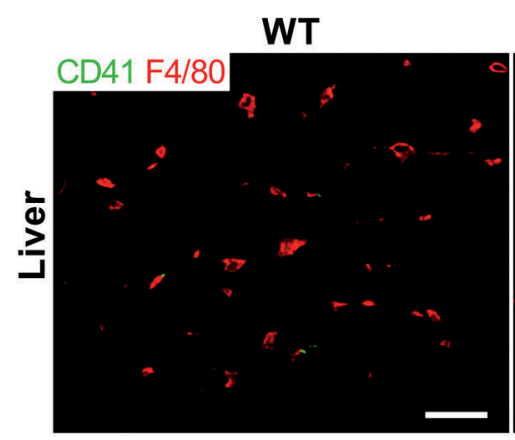

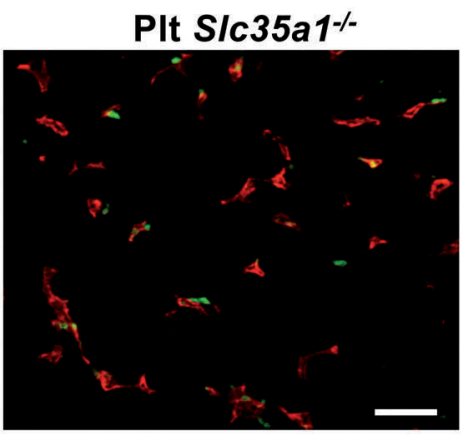

B

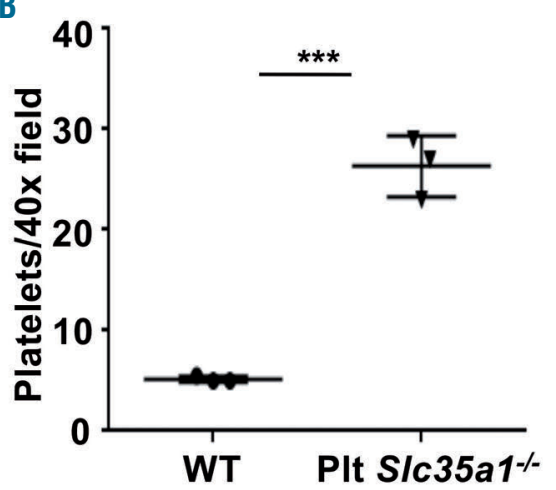

Figure 8. Increased clearance of desialylated platelets by Küpffer cells in PIt Slc35a1 type (WT) $(n=4)$ and PIt S/c35a1 ${ }^{--}(n=3)$ mice stained with anti-F4/80 (red) and anti-CD41 (green) antibodies. Scale bar, $25 \mu \mathrm{m}$. (B) Quantification of WT ( $=3$ mice) and S/c35a1 $1^{-/-}(n=3$ mice) platelets in the liver per image (40X). Data are means \pm standard deviation. ***P<0.001.

regulate the specific sialylation pattern of cells, many of them have overlapping functions. Thus, it is difficult to determine the overall biological role of sialylation in a given specific cell type. However, the CST, encoded by Slc35a1, is essential to transport CMP-SA from the cytosol into the Golgi apparatus for the sialylation process. ${ }^{39}$ Therefore, we generated conditional Slc35atff mice for cell-specific deletion of sialylation. Sialylation is the major capping glycan structure on platelet membrane glycoproteins. Although significant progress has been made recent$\mathrm{ly}_{,}{ }^{11}$ the biological function of total sialylation on megakaryocytes and platelets is not yet fully understood. Moreover, patients with sialylation defects, such as SLC35A1-CDG, exhibited macrothrombocytopenia in common. ${ }^{20-23,40}$ To investigate this issue, we generated a Plt

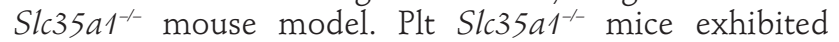
impaired megakaryocytopoiesis, impaired megakaryocyte maturation, and excessive platelet clearance in the liver, indicating that sialylation is essential for both platelet generation and clearance.

Platelets express several sialyltransferases, such as ST3Gal-I and ST3Gal-IV. ${ }^{18,19,41}$ Macrothrombocytopenia is

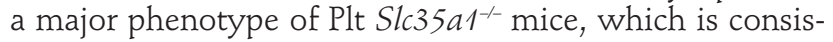
tent with mice lacking ST3Gal-I or ST3Gal-IV..$^{17,41,42}$ These data indicate that sialylation is critical for platelet homeo-

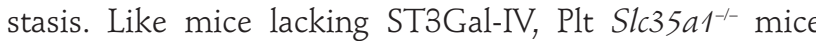
showed increased clearance of platelets in the liver. However, we did not detect significant numbers of desialylated platelets co-localized with hepatocytes as reported in ST3Gal-IV-deficient mice. ${ }^{17}$ Instead, we found that desialylated platelets were primarily co-localized with Küpffer cells in the liver. This result is consistent with our previous publication, ${ }^{7}$ indicating that increased clearance mediated by the Küpffer cells in the liver contributes to

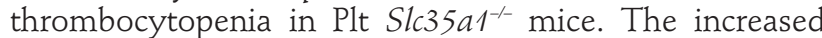
clearance of desialylated platelets in the liver and reduced numbers of megakaryocytes in the bone marrow found in Plt Slc35a1 $1^{-1}$ mice was not found in mice lacking ST3GalI, which have normal platelet clearance and normal megakaryocyte number, as reported in an abstract published in 2014. ${ }^{42}$ This discrepancy might be caused by redundant functions of different sialyltransferases in platelets. However, further studies comparing different mouse models directly are required to provide more insights into how sialylation regulates the homeostasis of megakaryocytes/platelets.

Thrombopoietin and its receptor $\mathrm{Mpl}$ are major regulators of megakaryocytopoiesis, megakaryocyte maturation, and platelet production. ${ }^{14,43}$ Our western blot analysis showed that $\mathrm{Mpl}$ expression was reduced in Slc35a1-1- 
megakaryocytes, and quantitative reverse transcription PCR revealed no difference in $\mathrm{Mpl}$ transcripts between WT and Plt Slc35a 1 $^{-1}$ megakaryocytes, suggesting reduced

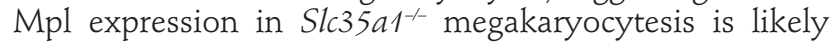
caused by impaired protein stability. Deficiency of $\mathrm{Mpl}$ may contribute to the defective platelet production as the percentage of proplatelet-forming megakaryocytes and absolute count of reticulated platelets were significantly decreased in Plt Slc35a1-1 mice, although we did not reproducibly detect impaired Mpl-mediated signaling in

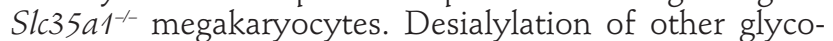
proteins, such as GPIbo, on megakaryocytes and platelets may also contribute to reduced platelet production. Previous studies have shown that refrigeration-mediated desialylation in platelets results in GPIb $\alpha$ shedding in vitro..$^{44,45}$ In our study, while both Slc35a ${ }^{-1}$ megakaryocytes and platelets exhibited significant reductions of sialylation, GPIb $\alpha$ level was only decreased on Slc35a1-1platelets, while it remained unchanged on Slc35a1-1 megakaryocytes. These results support that reduced sialylation causes shedding of GPIb $\alpha$ on circulating platelets, likely due to the action of plasma proteases. However, as

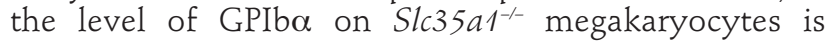
unchanged, it less likely to contribute to reduced platelet generation. Nevertheless, whether desialylation directly affects the function of these surface glycoproteins needs further study.

Genetic mutations of the SLC35A1 gene cause a rare form of a congenital disorder of glycosylation, SLC35A1CDG. So far, only three human cases associated with SLC35A1 mutations have been reported. A recent study of such a patient showed that CST is not required for proplatelet formation, ${ }^{23}$ a finding that differs from our mouse

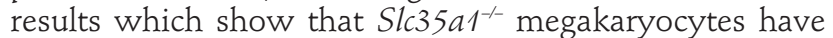
defective proplatelet formation. There are a few differences that may contribute to some of the inconsistencies between the study of the human patient and our mouse model, including: (i) the SLC35A1-CDG patient has a point mutation in the transmembrane domain, which might still have some residual CMP-SA transporting activities; (ii) in vitro-differentiated megakaryocytes from isolated CD34+ cells from the patient's peripheral blood were used in the human study while bone marrow megakaryocytes were used in our study; (iii) the SLC35A1-CDG patient has reduced sialylation in all cell types while our mouse model has deletion of Slc35a1 specifically in megakaryocytes and platelets, and (iv) potential differences between human and mouse megakaryocytes. Nevertheless, we believe our mouse model, which is the first mouse model of Slc35a1 deficiency in megakaryocytes and platelets, will be valuable for determining the role of sialylation in platelet biology and helping to dissect the molecular and cellular pathogenesis of thrombocytopenia, a primary clinical presentation of patients with SLC35A1-CDG. The study of patients and the mouse model are complementary.

In addition to SLC35A1-CDG, desialylation contributes to many acquired platelet disorders, such as refractory immune thrombocytopenia and prolonged isolated thrombocytopenia after hematopoietic stem cell transplantation. ${ }^{45,46}$ Our current and future studies using the Plt

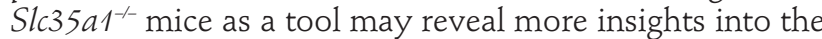
pathogenesis of these platelet problems, which may in turn lead to new diagnoses and therapies.

\section{Disclosures}

This study is a collaboration between Dr. Changgeng Ruan's laboratory at Jiangsu Institute of Hematology (JIH), Soochow University, China, and the Oklahoma Medical Research Foundation (OMRF), USA. The study was funded by JIH and $O M R F$. There are no intellectual conflicts of interests.

\section{Contributions}

$X M, Y L, Y K, J H, S A A$ and PA designed and conducted experiments, analyzed data, and drafted the manuscript. HS, $Y K, Y J$, and $X B$ contributed to manuscript preparation. JF, CR and LX designed the study, analyzed data, and wrote the paper.

\section{Funding}

This study was supported by grants from the National Natural Science Foundation of China (81520108005,81470825, 30928010, 81370617, 81800114), Jiangsu Provincial Key Medical Center (ZX201102), Jiangsu Provincial Special Program of Medical Science (BL2012005), and the Priority Academic Program Development (PAPD) of Jiangsu Higher Education Institutions (YXZXA2016002), National Institutes of Health (IS10OD018530, P41GM10349010), and a research grant from Oklahoma Medical Research Foundation.

\section{References}

1. de Gaetano G. Historical overview of the role of platelets in hemostasis and thrombosis. Haematologica. 2001;86(4):349-356.

2. Bryckaert M, Rosa JP, Denis CV, Lenting PJ. Of von Willebrand factor and platelets. Cell Mol Life Sci. 2015;72(2):307-326.

3. Herzog BH, Fu J, Wilson SJ, et al. Podoplanin maintains high endothelial venule integrity by interacting with platelet CLEC-2. Nature. 2013;502(7469):105-109.

4. Holinstat M. Normal platelet function. Cancer Metastasis Rev. 2017;36(2):195-198.

5. Mauler M, Bode C, Duerschmied D. Platelet serotonin modulates immune functions. Hamostaseologie. 2016;36(1):11-16.

6. Nurden AT. Platelet membrane glycoproteins: a historical review. Semin Thromb Hemost. 2014;40(5):577-584.

7. Li Y, Fu J, Ling Y, et al. Sialylation on O-gly- cans protects platelets from clearance by liver Kupffer cells. Proc Natl Acad Sci U S A. 2017;114(31):8360-8365.

8. Schauer R. Sialic acids as regulators of molecular and cellular interactions. Curr Opin Struct Biol. 2009;19(5):507-514.

9. Chen X, Varki A. Advances in the biology and chemistry of sialic acids. ACS Chem Biol. 2010;5(2):163-176.

10. Chen W, Druzak SA, Wang Y, et al. Refrigeration-induced binding of von Willebrand factor facilitates fast clearance of refrigerated platelets. Arterioscler Thromb Vasc Biol. 2017;37(12):2271-2279

11. Grozovsky R, Giannini S, Falet $H$, Hoffmeister KM. Regulating billions of blood platelets: glycans and beyond. Blood. 2015;126(16):1877-1884.

12. Grewal PK, Uchiyama S, Ditto D, et al. The Ashwell receptor mitigates the lethal coagulopathy of sepsis. Nat Med. 2008;14(6):648655.
13. Rumjantseva V, Grewal PK, Wandall HH, et al. Dual roles for hepatic lectin receptors in the clearance of chilled platelets. Nat Med. 2009;15(11):1273-1280.

14. Grozovsky R, Begonja AJ, Liu K, et al. The Ashwell-Morell receptor regulates hepatic thrombopoietin production via JAK2-STAT3 signaling. Nat Med. 2015;21(1):47-54

15. Wang Y, Jobe SM, Ding X, et al. Platelet biogenesis and functions require correct protein O-glycosylation. Proc Natl Acad Sci U S A. 2012;109(40):16143-16148.

16. Kudo T, Sato T, Hagiwara K, et al. C1galt1deficient mice exhibit thrombocytopenia due to abnormal terminal differentiation of megakaryocytes. Blood. 2013;122(9):16491657

17. Sorensen AL, Rumjantseva V, NayebHashemi S, et al. Role of sialic acid for platelet life span: exposure of beta-galactose results in the rapid clearance of platelets from the circulation by asialoglycoprotein 
receptor-expressing liver macrophages and hepatocytes. Blood. 2009;114(8):1645-1654.

18. Lee-Sundlov MM, Ashline DJ, Hanneman AJ, et al. Circulating blood and platelets supply glycosyltransferases that enable extrinsic extracellular glycosylation. Glycobiology. 2017;27(2):188-198.

19. Wandall HH, Rumjantseva V, Sorensen AL, et al. The origin and function of platelet glycosyltransferases. Blood. 2012;120(3):626635.

20. Willig TB, Breton-Gorius J, Elbim C, et al. Macrothrombocytopenia with abnormal demarcation membranes in megakaryocytes and neutropenia with a complete lack of sialyl-Lewis-X antigen in leukocytes--a new syndrome? Blood. 2001;97(3):826-828.

21. Martinez-Duncker I, Dupre T, Piller V, et al. Genetic complementation reveals a novel human congenital disorder of glycosylation of type II, due to inactivation of the Golgi CMP-sialic acid transporter. Blood. 2005;105(7):2671-2676.

22. Mohamed M, Ashikov A, Guillard M, et al. Intellectual disability and bleeding diathesis due to deficient CMP--sialic acid transport. Neurology. 2013;81(7):681-687.

23. Kauskot A, Pascreau T, Adam F, et al. A mutation in the gene coding for the sialic acid transporter SLC35A1 is required for platelet life span but not proplatelet formation. Haematologica. 2018;103(12):e613e617.

24. Nji E, Gulati A, Qureshi AA, et al. Structural basis for the delivery of activated sialic acid into Golgi for sialylation. Nat Struct Mol Biol. 2019;26(6):415-423.

25. Fu J, Gerhardt H, McDaniel JM, et al. Endothelial cell O-glycan deficiency causes blood/lymphatic misconnections and consequent fatty liver disease in mice. J Clin Invest. 2008;118(11):3725-3737.

26. Pan Y, Yago T, Fu J, et al. Podoplanin requires sialylated O-glycans for stable expression on lymphatic endothelial cells and for interaction with platelets. Blood. 2014;124(24): 3656-3665

27. Herisson F, Frodermann V, Courties G, et al.
Direct vascular channels connect skull bone marrow and the brain surface enabling myeloid cell migration. Nat Neurosci. 2018;21(9):1209-1217.

28. Jones MB, Oswald DM, Joshi S, et al. B-cellindependent sialylation of IgG. Proc Natl Acad Sci U S A. 2016;113(26):7207-7212.

29. Nagel T, Meyer B. Simultaneous characterization of sequence polymorphisms, glycosylation and phosphorylation of fibrinogen in a direct analysis by LC-MS. Biochim Biophys Acta. 2014;1844(12):2284-2289.

30. George JN. Platelet IgG: measurement, interpretation, and clinical significance. Prog Hemost Thromb. 1991;10:97-126.

31. George JN, Saucerman S. Platelet IgG, IgA $\mathrm{IgM}$, and albumin: correlation of platelet and plasma concentrations in normal subjects and in patients with ITP or dysproteinemia. Blood. 1988;72(1):362-365.

32. Michelson AD, Cattaneo M, Frelinger A, et al. (editors). Platelets (4th edition). Elsevier 2019; 79-97.

33. Bergmeier W, Piffath CL, Cheng G, et al. Tumor necrosis factor-alpha-converting enzyme (ADAM17) mediates GPIbalpha shedding from platelets in vitro and in vivo. Circ Res. 2004;95(7):677-683.

34. Jansen AJ, Josefsson EC, Rumjantseva V, et al. Desialylation accelerates platelet clearance after refrigeration and initiates GPIb $\alpha$ metalloproteinase-mediated cleavage in mice. Blood. 2012;119(5):1263-1273.

35. Draper JE, Sroczynska P, Leong HS, et al. Mouse RUNX1C regulates premegakaryocytic/erythroid output and maintains survival of megakaryocyte progenitors. Blood. 2017;130(3):271-284.

36. Draper JE, Sroczynska P, Tsoulaki O, et al. RUNX1B expression is highly heterogeneous and distinguishes megakaryocytic and erythroid lineage fate in adult mouse hematopoiesis. PLoS Genet. 2016;12(1): e1005814.

37. Albu RI, Constantinescu SN. Extracellular domain N-glycosylation controls human thrombopoietin receptor cell surface levels. Front Endocrinol (Lausanne). 2011;2:71.
38. Sorensen AL, Hoffmeister KM, Wandall HH Glycans and glycosylation of platelets: current concepts and implications for transfusion. Curr Opin Hematol. 2008;15(6):606611.

39. Eckhardt $M$, Muhlenhoff $M$, Bethe $A$, Gerardy-Schahn R. Expression cloning of the Golgi CMP-sialic acid transporter. Proc Natl Acad Sci U S A. 1996;93(15):7572-7576.

40. Jones C, Denecke J, Strater R, et al. A novel type of macrothrombocytopenia associated with a defect in alpha2,3-sialylation. Am J Pathol. 2011;179(4):1969-1977.

41. Ellies LG, Ditto D, Levy GG, et al Sialyltransferase ST3Gal-IV operates as a dominant modifier of hemostasis by concealing asialoglycoprotein receptor ligands. Proc Natl Acad Sci U S A. 2002;99(15): 10042-10047.

42. Grozovsky R, Giannini S, Ramsey H, SolaVisner M, Hoffmeister KM. T-antigen expression causes macrothrombocytopenia and extensive hemophagocytosis in mice lacking the sialyltransferase ST3Gal-I specifically in megakaryocytes. Blood. 2014;124 (21):94.

43. Alexander WS, Roberts AW, Nicola NA, L $\mathrm{R}$, Metcalf D. Deficiencies in progenitor cells of multiple hematopoietic lineages and defective megakaryocytopoiesis in mice lacking the thrombopoietic receptor c-Mpl. Blood. 1996;87(6):2162-2170.

44. Jansen AJ, Josefsson EC, Rumjantseva V, et al. Desialylation accelerates platelet clearance after refrigeration and initiates GPIbalpha metalloproteinase-mediated cleavage in mice. Blood. 2012;119(5):1263-1273.

45. Li J, van der Wal DE, Zhu G, et al Desialylation is a mechanism of Fc-independent platelet clearance and a therapeutic target in immune thrombocytopenia. Nat Commun. 2015;6:7737.

46. Zhang XH, Wang OM, Zhang JM, et al. Desialylation is associated with apoptosis and phagocytosis of platelets in patients with prolonged isolated thrombocytopenia after allo-HSCT. J Hematol Oncol. 2015; $8: 116$ 